\title{
Correlative Imaging of Stacking Faults using Atom Probe Tomography (APT) and Scanning Transmission Electron Microscopy (STEM)
}

\author{
S. Dumpala ${ }^{1}$, A. A. Oni ${ }^{2}$, S. Padalkar ${ }^{1}$, S.R. Broderick ${ }^{1}$, J. M. LeBeau ${ }^{2}$ and K. Rajan ${ }^{1}$ \\ ${ }^{1 .}$ Department of Materials Science and Engineering and Institute for Combinatorial Discovery, \\ Iowa State University, 2220 Hoover Hall, Iowa State University, Ames, IA 50011, \\ krajan@iastate.edu \\ ${ }^{2}$ Department of Materials Science and Engineering, North Carolina State University, Raleigh, \\ NC, 27695
}

Doping in Ni3Al alloy systems can have a significant enhancement in various properties including high temperature strength, fatigue and creep. The enhanced high temperature mechanical properties of Ni-based superalloys are attributed to microstructure consisting of $\gamma^{\prime}$ matrix strengthened by ordered $\gamma^{\prime}$ precipitates [1]. Segregation or partitioning of the doping elements in these two phases plays a vital role in mechanical properties. Thus correlative atomistic scale chemical imaging techniques are developed for better understanding of these microstructural changes and their effect on the properties.

Experimental analyses of STEM and APT microscopy have been performed on Ni-based alloys. The Ni-Al-Cr system showed interesting behavior, with the STEM and atomic-resolution EDS results clearly imaging stacking faults and segregation behavior of the $\mathrm{Cr}$ to the disordered phase. The elemental maps of the superalloy in the $\gamma$ ' show that Al and Cr occupy the same atomic column. Correlative APT studies were performed where $\mathrm{Cr}$ segregation was observed in agreement with STEM data. From the APT reconstructed image, it is also noticed that $\mathrm{Al}$ and $\mathrm{Cr}$ occupy similar positions. By capturing the same microstructural changes and chemical segregation between APT and STEM, we now have developed an enhanced approach for correlative microscopy, linking the structural and defect analyses of STEM with chemical analysis at the interface with APT.

\section{References:}

[1] D. Blavette et al, Microscopy and Microanalysis 13 (2007), 06

[2] The authors acknowledge support from Air Force Office of Scientific Research grants:

FA9550-11-1-0158 and FA9550-12-0496; and NSF grant: ARI Program CMMI-09-389018. 

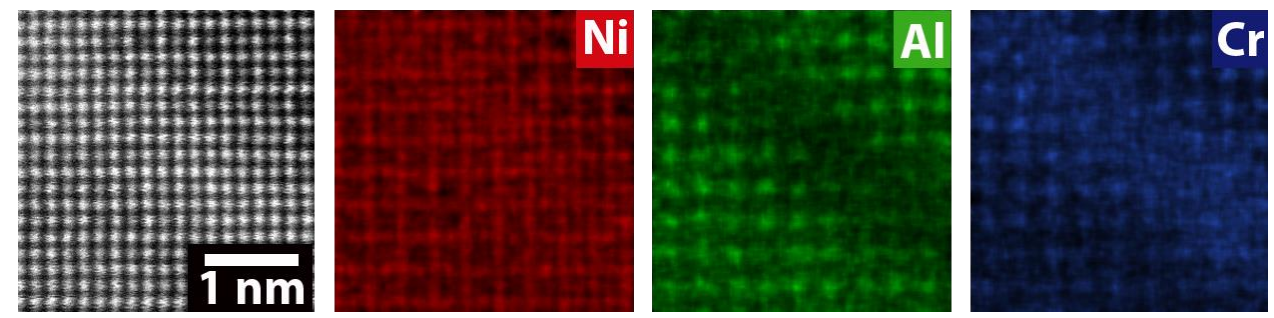

Figure 1. Atomic resolution EDS maps across the $\gamma / \gamma^{\prime}$ interfaces of the NiAlCr superalloy in the $<100>$ projection. The elemental maps show that $\mathrm{Cr}$ preferentially partitions to the $\gamma$ phase, and occupies the $\mathrm{Al}$ sites in the intermetallic phase.
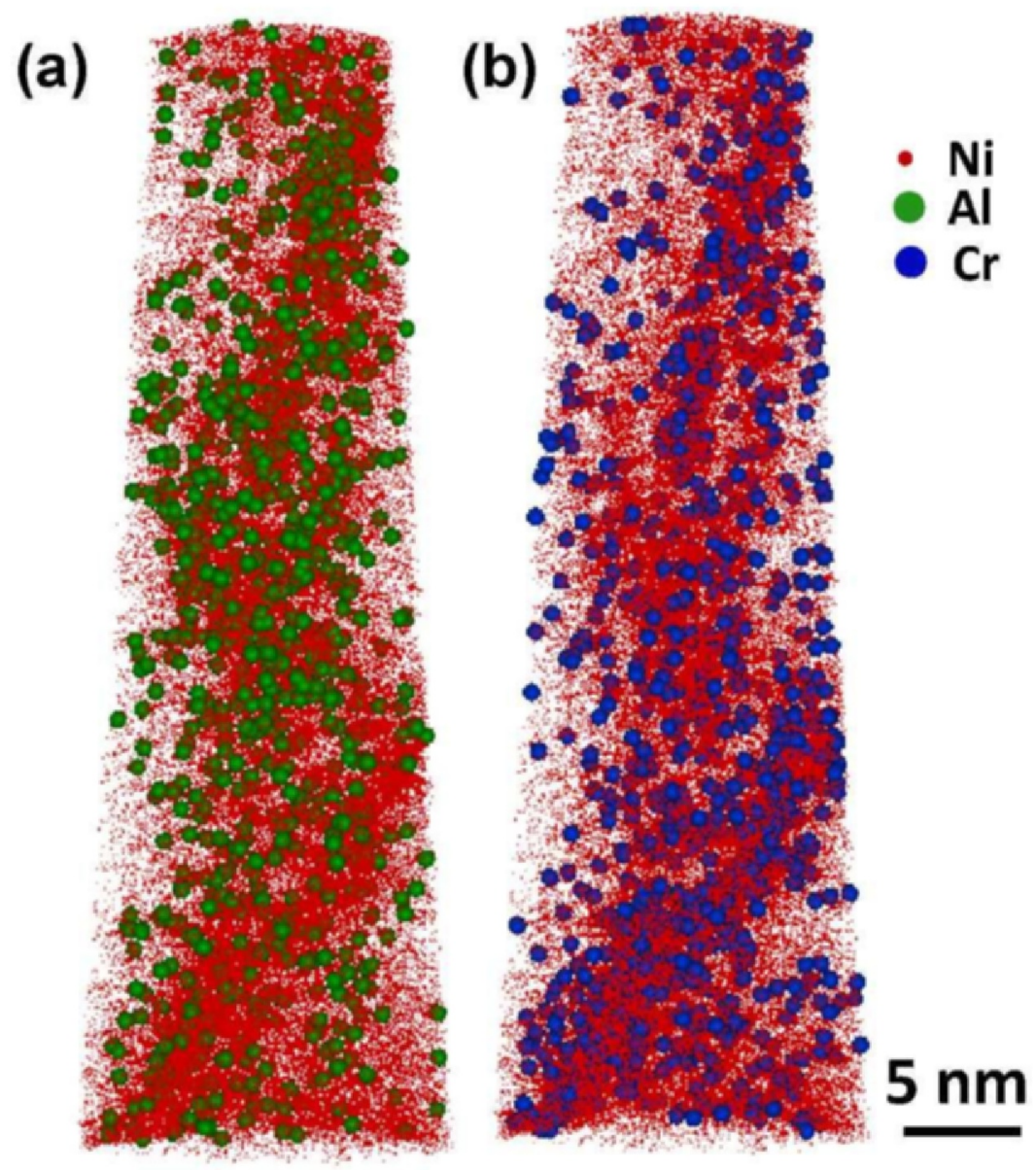

Figure 2. 3D atom probe reconstructed images of same Ni-Al-Cr alloy with (a) Aluminum and (b) Chromium atoms highlighted, respectively. Red atoms are $\mathrm{Ni}$, green atoms are $\mathrm{Al}$ and blue atoms are $\mathrm{Cr}$. 